Collectanea Mathematica (electronic version): http://www.imub.ub.es/collect

Collect. Math. 60, 1 (2009), 79-88

(C) 2009 Universitat de Barcelona

\title{
Ball proximinality of equable spaces
}

\author{
S. LALithambigai \\ Department of Mathematics, Pondicherry University Kalapet, \\ Pondicherry-605014, India \\ E-mail: S_lalithambigai@yahoo.co.in \\ Received March 25, 2008. Revised August 19, 2008.
}

\begin{abstract}
The notion of ball proximinality and the strong ball proximinality were recently introduced in [2]. We prove that an equable subspace $Y$ of a Banach space $X$ is strongly ball proximinal and the metric projection from $X$, onto the closed unit ball of $Y$, is Hausdorff metric continuous.
\end{abstract}

\section{Introduction and notation}

Let $X$ be a normed linear space and $A$, any closed subset of $X$. We say $A$ is proximinal in $X$ if for every $x$ in $X$, the set

$$
P_{A}(x)=\{y \in A:\|x-y\|=d(x, A)\}
$$

is a non-empty set.

The notion of ball proximinality of a closed subspace was introduced in [2], motivated by the example given in [10].

Definition 1.1 A subspace $Y$ of a normed linear space $X$ is ball proximinal in $X$ if $Y_{1}$, the closed unit ball of $Y$, is proximinal in $X$.

It is easily verified (see $[10,2]$ ) that $Y$ is ball proximinal in $X$, then $Y$ is proximinal in $X$. That the converse is not true, was shown in [10] by a counter example. Thus, ball proximinality implies proximinality, while the converse is not true.

One of the well known and often used method, to prove proximinality of a closed set or a subspace $C$ in $X$, is to construct a Cauchy minimizing sequence from $C$, for

Keywords: Proximinal, Ball proximinal, Strongly ball proximinal, Metric projection, Hausdorff metric continuous.

MSC2000: 46B20; 41A50; 41A65. 
each element of $X$ to be approximated. In particular for subspaces, such constructions are usually made using a suitable intersection property of balls that may be available. For example, the proximinality of M-ideals is proved by using the 3-ball intersection property of the M-ideals.

The other intersection property of balls, which facilitates construction of Cauchy minimizing sequences, is a property that has found frequent mention in the years from 1968-1980, in articles [8, 7, 11, 6], of Olech, Mach, Yost and Lau respectively. This property (or a mild variation of it) is called by different names in the above mentioned references and we follow the nomenclature, viz equability, used by Yost in [11]. This notion of equability easily renders itself to be a sufficient condition for ball proximinality and in fact, results in a stronger notion of ball proximinality, namely, strong ball proximinality. Further, the Hausdorff metric continuity of the metric projection onto the closed unit ball of an "equable" subspace follows as a consequence of the equability criteria.

We use the following notation and definitions in this paper. If $X$ is a normed linear space, let $X_{1}=\{x \in X:\|x\| \leq 1\}$, the closed unit ball of $X$. For $x$ in $X$ and $r>0$, we set

$$
B(x, r)=\{y \in X:\|x-y\| \leq r\}, \quad D(x, r)=\{y \in X:\|x-y\|<r\}
$$

and if $A$ is a subset of $X$ then the distance of $x$ from the set $A$ is denoted by $d(x, A)$. That is,

$$
d(x, A)=\inf \{\|x-z\|: z \in A\} .
$$

If $A$ and $B$ are bounded, nonempty subsets of a Banach space, we denote by $d_{H}(A, B)$ the Hausdorff metric distance between $A$ and $B$, given by

$$
d_{H}(A, B)=\max \left\{\sup _{a \in A} d(a, B), \sup _{b \in B} d(b, A)\right\} .
$$

For any $\delta>0$ we set

$$
P_{A}(x, \delta)=\{z \in A:\|x-z\|<d(x, A)+\delta\} .
$$

Following [3], we say a proximinal set $A$ of a normed linear space $X$ is strongly proximinal if for each $x$ in $X$ and $\epsilon>0$, there exists $\delta>0$ such that

$$
s(x, \delta)=\sup \left\{d\left(z, P_{A}(x)\right): z \in P_{A}(x, \delta)\right\}<\epsilon .
$$

Definition 1.2 A ball proximinal subspace $Y$ of $X$ is called strongly ball proximinal, if $Y_{1}$ is strongly proximinal in $X$.

In Section 2 of this paper, we prove that equable subspaces (Definition 2.1 below) are strongly ball proximinal and the corresponding metric projection is Hausdorff metric continuous. In Section 3 we apply these results and derive, as corollaries, the main results of [5]. It is to be mentioned here that our proofs of these results are brief and simpler than that of [5]. Also, we produce many new examples of ball proximinal spaces, using one of our main results (Theorem 2.6). In Section 4, we show that equability is stable under the infinite $c_{0}$ - direct sums and present an example of a Banach space $X$ that is not strongly ball proximinal in itself. 


\section{Main results}

We need the following notation and definitions in the sequel.

For any normed linear space $X$, let $\mathcal{C}(X)$ denotes the class of all closed bounded subsets of $X$. We equip $\mathcal{C}(X)$ with the Hausdorff metric $d_{H}$ given by (1.1). Let $\mathcal{D}$ be subset of $\mathcal{C}(X)$ and let $F$ be a map from $\mathcal{D}$ into $\mathcal{C}(X)$. We say $F$ is continuous if $F$ is continuous as map from $\left(\mathcal{D}, d_{H}\right)$ into $\left(\mathcal{C}(X), d_{H}\right)$.

Let $A$ be a proximinal subset of a normed linear space $X$. Then the map $P_{A}$ : $X \rightarrow \mathcal{C}(A)$ given by (1.1), is called the metric projection from $X$ onto $A$. We say the set valued map $P_{A}$ is Hausdorff metric continuous (H.m.c) if $P_{A}$ is continuous as a map from the normed linear space $X$ into the metric space $\left(\mathcal{C}(A), d_{H}\right)$.

Let $X$ and $Y$ be Banach spaces. By $C(Q, X)$, we denote the space of continuous functions from the compact Hausdorff space $Q$ into $X, l_{\infty}(\Gamma, X)$ denotes the space of bounded functions from the set $\Gamma$ into $X, K(X, Y)$ denotes the space of compact operators from $X$ into $Y$ and $L(X, Y)$ denotes the space of bounded linear operators from $X$ into $Y$.

In the rest of this section, we use the following notation. If $S$ is a compact Hausdorff space and $\Gamma$ is a non-empty set, $\phi: \Gamma \rightarrow S$ would denote a surjection. If $Q$ and $S$ are compact, Hausdorff spaces, then $\phi: Q \rightarrow S$ would denote a continuous surjection. Further, $T_{\phi}: C(S, X) \rightarrow l_{\infty}(\Gamma, X)(C(Q, X))$ would denote the map $T_{\phi}(f)=f \circ \phi$ for $f \in C(S, X)$.

Below, we list the definitions, and modifications of definitions from [7, 11], that are needed in the sequel.

Let $X$ be a Banach space and $Y$ be a subspace of $X$. Let $F$ be a bounded subset of $X$. For $x$ and $y$ in $X$, let $[x, y]$ denote the line segment between $x$ and $y$. That is $[x, y]=\{\lambda x+(1-\lambda) y: 0 \leq \lambda \leq 1\}$. Define

$$
\bar{r}_{F}=\inf \left\{r>0: F \subset B(y, r) \text { for some } \mathrm{y} \text { in } \mathrm{Y}_{1}\right\} .
$$

We define the map $H_{Y_{1}}$ from $\mathcal{C}(X)$ into $\mathcal{C}\left(Y_{1}\right)$ by

$$
H_{Y_{1}}(F)=\left\{y \in Y_{1}: F \subset B\left(y, \bar{r}_{F}\right)\right\}
$$

and for $\eta>0$

$$
H_{Y_{1}}(F, \eta)=\left\{y \in Y_{1}: F \subset B\left(y, \bar{r}_{F}(1+\eta)\right)\right\} .
$$

The following definition is central to our discussion. As mentioned earlier, variations of this definition had appeared in $[8,7,11,6]$. we use the terminology of Yost in [11].

Definition 2.1 Let $X$ be a Banach space and $Y$ be subspace of $X$. We say $Y$ is an equable subspace of $X$ if for every $\epsilon>0$, there is a $\delta>0$ and a map $\psi_{\epsilon}: Y \rightarrow[0,1]$ such that for every $y$ in $Y,\left\|y-\psi_{\epsilon}(y) y\right\| \leq \epsilon$ and $B(0,1) \cap B(y, 1+\delta) \subset B\left(\psi_{\epsilon}(y) y, 1\right)$.

It has been shown in Yost [11] that $\delta(\epsilon)$ tends to zero as $\epsilon$ tends to zero and so we assume that $\delta(\epsilon) \leq 1$, for every $\epsilon>0$.

A subspace $Y$ of $X$ is called a very equable subspace of $X$, if $Y$ is an equable subspace of $X$ and for each $\epsilon>0$ the map $\psi_{\epsilon}$ is continuous. If $X$ is an (a very) equable subspace of $X$, we simply say that $X$ is an (a very) equable space. 
Remark 2.2 Definition 2.1 is a modification of the definition of equable spaces, as given in Yost [11]. The requirement that the element $\Psi_{\epsilon}(y)$ is in $[0, y]$ is essential for our purpose and we have incorporated this in our definition. However, every one of the spaces that are equable according to Yost and cited in [11], satisfy this additional requirement and thus satisfy our definition of equability.

\section{Proposition A [11]}

$X$ is uniformly convex if and only if $X$ is equable and strictly convex.

We list below the examples of equable subspaces given in [11].

\section{Theorem B [11]}

Let $X$ be a Banach space.

1. If $X$ is an equable Banach space with an equability map $\psi_{\epsilon}$ and $Y$ is a subspace of $l_{\infty}(\Gamma, X)$ which is invariant under each $\psi_{\epsilon}$, then $Y$ is an equable subspace of $l_{\infty}(\Gamma, X)$.

2. If $X$ is very equable and $S$ is compact Hausdorff space, then $T_{\phi}(C(S, X))$ is a very equable subspace of $l_{\infty}(\Gamma, X)$.

3. If $Q$ and $S$ are compact Hausdorff spaces and $X$ is very equable Banach space, then $T_{\phi}(C(S, X))$ is an equable subspace of $C(Q, X)$.

\section{Theorem C [11]}

In each of the following cases, $K(X, Y)$ is an equable subspace of $L(X, Y)$.

1. For any Banach space $X$ with $X^{*}$ is very equable and $Y=C(Q), Q$ is compact Hausdorff space.

2. $X$ is uniformly smooth and $Y=C(Q)$.

3. $X=L_{1}(\mu)$ and $Y=C(Q)$.

4. $X=L_{1}(\mu)$ and $Y$ is very equable.

5. $X=L_{1}(\mu)$ and $Y$ is uniformly convex.

In the rest of the paper, we use the following notation. For $\epsilon>0$ and $y$ in $Y, \Psi_{\epsilon}(y)$ will denote the element $\psi_{\epsilon}(y) y$ in $Y$. Note that $\Psi_{\epsilon}(y)$ is in $[0, y]$ and $\left\|y-\Psi_{\epsilon}(y)\right\| \leq \epsilon$.

We make frequent use of the following remark, regarding application of the equability criteria, in the sequel.

Remark 2.3 Let $Y$ be an equable subspace of a Banach space $X$. Then for any real scalar $s$ and elements $y, z$ and $w$ in $Y$, using the equability of $Y$ (Definition 2.1) we have

$$
B\left(\frac{y}{s}, 1\right) \cap B\left(\frac{z}{s},(1+\delta)\right) \subset B\left(\frac{w}{s}, 1\right),
$$

where $w=s\left\{\Psi_{\epsilon}\left(\frac{z-y}{s}\right)+\frac{y}{s}\right\}$ and $\delta$ corresponds to $\epsilon$ in the definition of equability of $Y$. Note that

$$
\left\|\frac{z-w}{s}\right\|=\left\|\Psi_{\epsilon}\left(\frac{z-y}{s}\right)-\frac{z-y}{s}\right\| \leq \epsilon,
$$


and if $y$ and $z$ are in $Y_{1}$, then $\Psi_{\epsilon}\left(\frac{z-y}{s}\right)+\frac{y}{s}$ is in $\left[\frac{y}{s}, \frac{z}{s}\right]$ and hence $w=s\left\{\Psi_{\epsilon}\left(\frac{z-y}{s}\right)+\frac{y}{s}\right\}$ is in $Y_{1}$.

We now prove our main results. The proof of the following theorem is similar to that of [11, Proposition 1.7.1].

\section{Theorem 2.4}

Let $X$ be a Banach space, $Y$ an equable subspace of $X$. Then $H_{Y_{1}}(F)$ is a nonempty set, for every bounded subset $F \subset X$.

Proof. Let $F$ be a bounded subset of $X$. It is enough to show that there exists $y$ in $Y_{1}$ such that $\|x-y\| \leq \bar{r}_{F}$, for all $x$ is in $F$. Let $\epsilon>0$ be given. Put $r=\bar{r}_{F}$ and $\epsilon_{n}=\frac{\epsilon}{r 2^{n+1}}, n \in \mathbb{N}$ and let $\delta_{n}=\delta\left(\epsilon_{n}\right)$ corresponds to $\epsilon_{n}$, in the Definition 2.1 of equability. With out loss of generality, we assume that the sequence $\left\{\delta_{n}\right\}$ decreases to zero. We now inductively construct a sequence $\left\{y_{n}\right\} \subseteq Y_{1}$ with $\left\|y_{n}-y_{n+1}\right\| \leq \frac{\epsilon}{2^{n}}$ and $F \subset B\left(y_{n},\left(1+\delta_{n}\right) \bar{r}_{F}\right)$, for all $n \geq 1$.

For $r=\bar{r}_{F}$ and $\epsilon_{1}$, using the definition of $\bar{r}_{F}$, we can get $y$ and $y_{1}$ in $Y_{1}$ with $F \subset B\left(y,\left(1+\delta_{2}\right) r\right) \cap B\left(y_{1},\left(1+\delta_{1}\right) r\right)$. Hence

$$
\frac{F}{\left(1+\delta_{2}\right) r} \subset B\left(\frac{y}{\left(1+\delta_{2}\right) r}, 1\right) \cap B\left(\frac{y_{1}}{\left(1+\delta_{2}\right) r}, \frac{1+\delta_{1}}{1+\delta_{2}}\right) .
$$

Note that $\frac{1+\delta_{1}}{1+\delta_{2}} \leq 1+\delta_{1}$. If

$$
\frac{y_{2}}{\left(1+\delta_{2}\right) r}=\Psi_{\epsilon_{1}}\left(\frac{y_{1}-y}{\left(1+\delta_{2}\right) r}\right)+\frac{y}{\left(1+\delta_{2}\right) r},
$$

then by Remark $2.2, y_{2}$ is in $Y_{1}$,

$$
B\left(\frac{y}{\left(1+\delta_{2}\right) r}, 1\right) \cap B\left(\frac{y_{1}}{\left(1+\delta_{2}\right) r}, \frac{1+\delta_{1}}{1+\delta_{2}}\right) \subset B\left(\frac{y_{2}}{\left(1+\delta_{2}\right) r}, 1\right),
$$

and

$$
\left\|\frac{y_{1}}{\left(1+\delta_{2}\right) r}-\frac{y_{2}}{\left(1+\delta_{2}\right) r}\right\|=\left\|\frac{y_{1}-y}{\left(1+\delta_{2}\right) r}-\Psi_{\epsilon_{1}}\left(\frac{y_{1}-y}{\left(1+\delta_{2}\right) r}\right)\right\| \leq \epsilon_{1} .
$$

Hence

$$
\left\|y_{1}-y_{2}\right\| \leq \frac{\epsilon\left(1+\delta_{2}\right)}{4} \leq \frac{\epsilon}{2}
$$

and

$$
F \subset B\left(y,\left(1+\delta_{2}\right) r\right) \cap B\left(y_{1},\left(1+\delta_{1}\right) r\right) \subset B\left(y_{2},\left(1+\delta_{2}\right) r\right) .
$$

Assume now that for $n \in \mathbb{N}$, the elements $y_{1}, y_{2}, \ldots, y_{n} \in Y_{1}$ with $F \subset B\left(y_{i},(1+\right.$ $\left.\delta_{i}\right) r$ ), for $i=1,2, \ldots, n$ and $\left\|y_{i}-y_{i+1}\right\| \leq \frac{\epsilon}{2^{i}}$, for $i=1, \ldots, n-1$, have already been constructed. Now for $r=\bar{r}_{F}$ and $\epsilon_{n}$, using the definition of $\bar{r}_{F}$, get $z$ in $Y_{1}$ with $F \subset B\left(z,\left(1+\delta_{n+1}\right) r\right)$. Observe that $\frac{1+\delta_{n}}{1+\delta_{n+1}} \leq 1+\delta_{n}$. By Remark 2.2, there exists $y_{n+1}$ in $Y_{1}$ with

$$
F \subset B\left(y_{n},\left(1+\delta_{n}\right) r\right) \cap B\left(z,\left(1+\delta_{n+1}\right) r\right) \subset B\left(y_{n+1},\left(1+\delta_{n+1}\right) r\right),
$$


where

$$
y_{n+1}=r\left(1+\delta_{n+1}\right)\left\{\Psi_{\epsilon_{n}}\left(\frac{y_{n}-z}{\left(1+\delta_{n+1}\right) r}\right)+\frac{z}{\left(1+\delta_{n+1}\right) r}\right\}
$$

and $y_{n+1}$ is in $Y_{1}$ and

$$
\left\|y_{n}-y_{n+1}\right\| \leq \frac{\epsilon}{2^{n}}
$$

It is clear that $\left\{y_{n}\right\} \subseteq Y_{1}$ is Cauchy and let $\left\{y_{n}\right\}$ converges to, say, $y_{0}$. Clearly $y_{0}$ belongs to $Y_{1}$ and $\left\|y_{0}-x\right\| \leq \bar{r}_{F}$, for all $x$ in $F$. Hence $H_{Y_{1}}(F)$ is a non-empty set.

\section{Theorem 2.5}

If $Y$ is an equable subspace of $X$, then given $\epsilon>0$, there exists $\eta>0$ such that $\sup \left\{d\left(y, H_{Y_{1}}(F)\right): y \in H_{Y_{1}}(F, \eta)\right\}<\epsilon$, where $H_{Y_{1}}(F, \eta)$ is given by (2.1). Also the map $H_{Y_{1}}: \mathcal{C}(X) \rightarrow \mathcal{C}\left(Y_{1}\right)$ is continuous.

Proof. Let $F$ be a bounded subset of $X$ and $\epsilon>0$. Select $\eta=\delta(\epsilon)>0$ as in Definition 2.1. By Theorem 2.4, $H_{Y_{1}}(F)$ is non-empty. Pick any $y$ in $H_{Y_{1}}(F, \eta)$ and $y_{1}$ in $H_{Y_{1}}(F)$. Clearly $F \subset B\left(y_{1}, \bar{r}_{F}\right) \cap B\left(y, \bar{r}_{F}(1+\eta)\right)$. Using Remark 2.2, we have $F \subset B\left(y_{2}, \bar{r}_{F}\right)$, where $y_{2}=\bar{r}_{F}\left\{\Psi_{\epsilon}\left(\frac{y-y_{1}}{\bar{r}_{F}}\right)+\frac{y_{1}}{\bar{r}_{F}}\right\}$ and further, $y_{2}$ is in $Y_{1}$. Thus $y_{2}$ is in $H_{Y_{1}}(F)$ and also, $\left\|y-y_{2}\right\| \leq \epsilon$. It now follows that

$$
\sup \left\{d\left(y, H_{Y_{1}}(F)\right): y \in H_{Y_{1}}(F, \eta)\right\}<\epsilon .
$$

To show $H_{Y_{1}}$ is continuous, let $\epsilon>0$ be given and $F$ and $G$ be in $\mathcal{C}(X)$ with $d_{H}(G, F) \leq \eta \bar{r}_{F}$. Then $\bar{r}_{G} \leq \bar{r}_{F}(1+\eta)$ and hence $H_{Y_{1}}(G) \subseteq H_{Y_{1}}(F, \eta)$. Then by (2.2), $\sup \left\{d\left(y, H_{Y_{1}}(F)\right): y \in H_{Y_{1}}(G)\right\}<\epsilon$. Similar argument shows that $\sup \left\{d\left(y, H_{Y_{1}}(G)\right)\right.$ : $\left.y \in H_{Y_{1}}(F)\right\}<\epsilon$. Thus $d_{H}\left(H_{Y_{1}}(F), H_{Y_{1}}(G)\right) \leq \epsilon$ and hence $H_{Y_{1}}$ is continuous.

The theorem below is an immediate consequence of Theorems 2.4 and 2.5, in the special case when the set $F$ is a singleton set.

\section{Theorem 2.6}

Let $Y$ be an equable subspace of $X$. Then $Y$ is strongly ball proximinal in $X$ and the metric projection from $X$ onto the closed unit ball $Y_{1}$ is H.m.c.

It is easy to see that the two theorems below follow immediately from Theorem $B$ and Theorem 2.6.

\section{Theorem 2.7}

Let $X$ be an equable Banach space with an equability map $\psi_{\epsilon}$. If $Y$ is a subspace of $l_{\infty}(\Gamma, X)$ which is invariant under each $\psi_{\epsilon}$. Then $Y$ is strongly ball proximinal in $l_{\infty}(\Gamma, X)$ and the metric projection from $l_{\infty}(\Gamma, X)$ onto $Y_{1}$ is H.m.c. If $X$ is very equable and $S$ is compact Hausdorff space, then $Z=T_{\phi}(C(S, X))$ is strongly ball proximinal in $l_{\infty}(\Gamma, X)$ and the metric projection from $l_{\infty}(\Gamma, X)$ onto $Z_{1}$ is H.m.c.

\section{Theorem 2.8}

If $Q$ and $S$ are compact Hausdorff spaces and $X$ is very equable Banach space, then $Z=T_{\phi}(C(S, X))$ is strongly ball proximinal in $C(Q, X)$ and the metric projection from $C(Q, X)$ onto $Z_{1}$ is H.m.c. 
The theorem below easily follows from Theorem $C$ and Theorem 2.6.

\section{Theorem 2.9}

The space $K(X, Y)$ is strongly ball proximinal in $L(X, Y)$ and the metric projection from $L(X, Y)$ onto $K(X, Y)_{1}$ is H.m.c, where the pairs $(X, Y)$ are given by 1 to 5 of Theorem $C$.

Property $\left(P_{1}\right)$ and Property $\left(P_{2}\right)$ are defined in Mach [7] and Yost [11] has shown that these two properties imply equability and very equability respectively. We quote below one of Mach's results about Properties $P_{1}$ and $P_{2}$ and it is easily seen that Corollaries 2.10 and 2.11 given below follow as a consequence.

\section{Theorem D [7]}

Let $X$ be a Banach space.

1. If $X$ is a uniformly convex Banach space, $Y$ a closed convex subset of $X$. Then the pair $(X, Y)$ has the property $\left(P_{2}\right)$.

2. Let $S$ be a compact Hausdorff space and the pair $(X, Y)$ has the property $\left(P_{2}\right)$. Then the pair $\left(l_{\infty}(S, X), C(S, Y)\right)$, has the property $\left(P_{1}\right)$.

\section{Corollary 2.10}

Let $S$ be a compact Hausdorff space and the pair $(X, Y)$ has the property $\left(P_{2}\right)$. Then $C(S, Y)$ is strongly ball proximinal in $l_{\infty}(S, X)$ and the metric projection from $l_{\infty}(S, X)$ onto the closed unit ball $C(S, Y)_{1}$ is H.m.c.

\section{Corollary 2.11}

If $X$ is uniformly convex Banach space, $Y$ a closed convex subset of $X$ and $S$ a Hausdorff space then $C(S, Y)$ is strongly ball proximinal in $l_{\infty}(S, X)$ and the metric projection from $l_{\infty}(S, X)$ onto the closed unit ball $C(S, Y)_{1}$ is H.m.c.

Let $Q$ be compact Hausdorff space. Yost [11] has shown that a closed * subalgebra of $C(Q)$ is a very equable subspace of $C(Q)$ and hence the following theorem, from [5], is an immediate consequence of Theorem 2.6.

\section{Theorem E [5]}

Every closed ${ }^{*}$ subalgebra $\mathcal{A}$ of $C(Q)$, where $Q$ is a compact Hausdorff space, is strongly ball proximinal and the metric projection from $C(Q)$ onto $\mathcal{A}_{1}$ is H.m.c.

Let $Q$ be compact Hausdorff space and $X$ be uniformly convex space. Let $M$ be any $C(Q)$ module in $C(Q, X)$. Since $X$ is uniformly convex space, there exists a continuous onto map $\phi: Q^{\prime} \rightarrow Q$, where $Q^{\prime}$ is the stone space of $C(Q)^{* *}$, such that the canonical embedding of $C(Q, X)$ in $C(Q, X)^{* *}$ is given by $f \rightarrow f \circ \phi$. In [9], T.S.S.R.K.Rao has shown that $M$ is proximinal in $C(Q, X)^{* *}$. However we observe that by 3$)$ of Theorem $B, M$ is an equable subspace of $C(Q, X)^{* *}$ and so is ball proximinal in $C(Q, X)^{* *}$. The following theorem is now an easy consequence of the Theorem 2.6. 


\section{Corollary 2.12}

Let $Q$ be a compact Hausdorff space and $X$ be uniformly convex space. Then any $C(Q)$ module $M$ in $C(Q, X)$ is strongly ball proximinal in $C(Q, X)^{* *}$ and the metric projection from $C(Q, X)^{* *}$ onto the closed unit ball of $M$ is H.m.c.

The following two results immediately follows from Corollary 2.12.

\section{Theorem F $[9]$}

Let $Q$ be compact Hausdorff space and $X$ be uniformly convex space. If $M$ is any $C(Q)$ module in $C(Q, X)$, then $M$ is proximinal in $C(Q, X)^{* *}$.

\section{Corollary 2.13}

Let $Q$ be a compact Hausdorff space and $X$ be uniformly convex space. Then $C(Q, X)$ is strongly ball proximinal in $C(Q, X)^{* *}$ and the metric projection from $C(Q, X)^{* *}$ onto the closed unit ball of $C(Q, X)$ is H.m.c.

\section{Stability}

\section{Theorem 3.1}

Let $X^{1}$ and $X^{2}$ be Banach spaces and $Y^{1}$ and $Y^{2}$ be equable subspaces of $X^{1}$ and $X^{2}$ respectively. Consider the following direct sums $X=X^{1} \oplus_{\infty} X^{2}$ and $Y=Y^{1} \oplus_{\infty} Y^{2}$. Then $Y$ is an equable subspace of $X$.

Proof. Let $\epsilon>0$ be given. Since $Y^{i}$ is an equable subspace of $X^{i}, i=1,2$, there is a $\delta_{i}>0$ and a function $\psi_{\epsilon}^{i}: Y^{i} \rightarrow[0,1], i=1,2$. such that for every $y_{i}$ in $Y^{i},\left\|y_{i}-\Psi_{\epsilon}^{i}\left(y_{i}\right)\right\| \leq \epsilon$ and $B(0,1) \cap B\left(y_{i}, 1+\delta_{i}\right) \subset B\left(\Psi_{\epsilon}^{i}\left(y_{i}\right), 1\right), i=1,2$. Choose $\delta=\min \left\{\delta_{1}, \delta_{2}\right\}, y=y_{1}+y_{2}$ and $\Psi_{\epsilon}(y)=\Psi_{\epsilon}^{1}\left(y_{1}\right)+\Psi_{\epsilon}^{2}\left(y_{2}\right)$. Then it is easily verified that $Y$ is an equable subspace of $X$ with equability map $\psi_{\epsilon}$.

\section{Theorem 3.2}

Let $\left\{X^{i}: i \in \mathbb{N}\right\}$ be a family of Banach spaces and $Y^{i}$ be equable subspace in $X^{i}$ for each $i \in \mathbb{N}$. Consider the following direct sums $X=\left(\oplus_{c_{0}} X^{i}\right)_{i \in \mathbb{N}}$ and $Y=\left(\oplus_{c_{0}} Y^{i}\right)_{i \in \mathbb{N}}$. Then $Y$ is an equable subspace of $X$.

Proof. Let $\epsilon>0$ be given and let $y=\left(y_{1}, y_{2}, \ldots\right)$ be in $Y$. Then there is $n_{0} \in \mathbb{N}$ such that $\left\|y_{i}\right\| \leq \epsilon$, for all $i>n_{0}$. Choose $\delta=\min \left\{\delta_{i}, 1 \leq i \leq n_{0}, \epsilon, i>n_{0}\right\}$, where $\delta_{i}, 1 \leq i \leq n_{0}$ are in the proof of Theorem 4.1. Let $z=y_{1}+y_{2}+\ldots$ and $\Psi_{\epsilon}(z)=\Psi_{\epsilon}^{1}\left(y_{1}\right)+\Psi_{\epsilon}^{2}\left(y_{2}\right)+\ldots$ Then it easily follows from Theorem 4.1 that $Y$ is an equable subspace of $X$.

\section{Example}

The author would like to express her thanks to Prof. G. Godefroy for indicating the construction of the following example. 
Construction of a Banach space which is not strongly ball proximinal in itself

It is an easy observation that normed linear spaces are ball proximinal in themselves. However the same does not hold for strongly ball proximinality, as shown in the example given below. Let $X$ be the sequence space $c_{0}$. Define

$$
\left\|\left|x\|\mid=\| x \|_{\infty}+\left(\sum_{n=1}^{\infty}\left(2^{-n} x_{n}\right)^{2}\right)^{1 / 2}\right.\right.
$$

where $x=\left(x_{1}, x_{2}, \ldots\right)$ is in $X$ and $\|x\|_{\infty}=\sup _{i \in \mathbb{N}}\left|x_{i}\right|$. Then $(X,\||\cdot \||)$ is strictly convex Banach space. Consider the element $z=(2,0,0, \ldots)$ in $X$. Then $\||z \||=3$ and $\frac{z}{\|z\| \|}=\left(\frac{2}{3}, 0,0, \ldots ..\right)$. Clearly $P_{X_{1}}(z)=\left\{\frac{z}{\|z\| \|}\right\}$ and

$$
d\left(z, X_{1}\right)=2=\left\|\left|z-\frac{z}{\||z \||} \|\right|\right.
$$

Let $\eta_{n}$ be sequence of positive numbers such that $\frac{1}{2^{n+1}}<\eta_{n}<\frac{1}{2^{n}}$. Let $z_{n}=$ $\left(y_{1}, y_{2}, \ldots ..\right)$, where $y_{i}=\left\{\begin{array}{ll}\frac{2}{3}-\eta_{i} & \text { if } \mathrm{i}=\{1, \mathrm{n}\} \\ 0 & \text { if } \quad \mathrm{i} \notin\{1, \mathrm{n}\}\end{array}\right.$.

Then

$$
\begin{aligned}
\left\|\left|z_{n} \|\right|\right. & =\frac{2}{3}-\eta_{n}+\left\{\left[\left(\frac{2}{3}-\eta_{n}\right)\left(\frac{1}{2}\right)\right]^{2}+\left[\left(\frac{2}{3}-\eta_{n}\right)\left(\frac{1}{2^{n}}\right)\right]^{2}\right\}^{1 / 2} \\
& \leq \frac{2}{3}-\eta_{n}+\frac{1}{2}\left(\frac{2}{3}-\eta_{n}\right)+\frac{1}{2^{n}}\left(\frac{2}{3}-\eta_{n}\right) \\
& \leq 1-\eta_{n}\left(\frac{3}{2}+\frac{1}{2^{n}}\right)+\frac{1}{2^{n}} \\
& \leq 1
\end{aligned}
$$

Also

$$
\begin{aligned}
\left\|\left|z-z_{n} \|\right|\right. & =\left\|\left|\left(\frac{4}{3}+\eta_{n}, 0,0, \ldots .0,-\left(\frac{2}{3}-\eta_{n}\right), 0, \ldots\right) \|\right|\right. \\
& =\frac{4}{3}+\eta_{n}+\left\{\left[\left(\frac{4}{3}+\eta_{n}\right)\left(\frac{1}{2}\right)\right]^{2}+\left[\left(\frac{2}{3}-\eta_{n}\right)\left(\frac{1}{2^{n}}\right)\right]^{2}\right\}^{1 / 2} \\
& \leq \frac{4}{3}+\eta_{n}+\left(\frac{4}{3}+\eta_{n}\right)\left(\frac{1}{2}\right)+\left(\frac{2}{3}-\eta_{n}\right)\left(\frac{1}{2^{n}}\right) \\
& \leq 2+\eta_{n}\left(\frac{3}{2}-\frac{1}{2^{n}}\right)+\frac{1}{2^{n}} .
\end{aligned}
$$

Hence $\lim _{n \rightarrow \infty}\left\|z_{n}-z\right\|=2=d\left(z, X_{1}\right)$. Now for any $n>1$, we have 


$$
\begin{aligned}
\left\|\frac{z}{\|z\| \|}-z_{n}\right\| \| & =\left\|\left(\eta_{n}, 0,0, \ldots .0,-\left(\frac{2}{3}-\eta_{n}\right), 0, \ldots\right)\right\| \| \\
& =\frac{2}{3}-\eta_{n}+\left\{\left(\frac{\eta_{n}}{2}\right)^{2}+\left[\left(\frac{2}{3}-\eta_{n}\right)\left(\frac{1}{2^{n}}\right)\right]^{2}\right\}^{1 / 2} \\
& \geq \frac{2}{3}-\eta_{n}+\frac{\eta_{n}}{2}-\left(\frac{2}{3}-\eta_{n}\right)\left(\frac{1}{2^{n}}\right) \\
& \geq \frac{1}{3}-\frac{\eta_{n}}{2}+\frac{\eta_{n}}{2} \\
& =\frac{1}{3} .
\end{aligned}
$$

It is now clear that $X_{1}$ is not strongly proximinal at $z$ and hence $X$ is not strongly ball proximinal in itself.

Acknowledgement. The author would like to thank Prof. V. Indumathi for guidance in the preparation of this paper. The author's research was supported by the CSIR Research Fellowship and she would like to thank the CSIR for their financial support.

\section{References}

1. Pr. Bandyopadhyay, B.-L. Lin, and T.S.S.R.K. Rao, Ball proximinality in Banach spaces, Banach spaces and their applications in analysis, 251-264, Conference in Honor of Nigel Kalton's $60^{\text {th }}$ Birthday, Berlin, 2007.

2. J. Blatter, Grothendieck Spaces in Approximation Theory, American Mathematical Society 120, Providence, 1972.

3. G. Godefroy and V. Indumathi, Strong proximinality and polyhedral spaces, Rev. Mat. Complut. 14 (2001), 105-125.

4. P. Harmand, D. Werner, and W. Werner, M-Ideals in Banach Spaces and Banach Algebras, Lecture Notes in Mathematics, 1574, Springer-Verlag, Berlin, 1993.

5. V. Indumathi, S. Lalithambigai, and B.-L. Lin, Ball proximinality of closed * subalgebras in $C(Q)$, Extracta Math. 22 (2007), 1-17.

6. K.S. Lau, Approximation by continuous vector-valued functions, Studia Math. 68 (1980), 291298.

7. J. Mach, Best simultaneous approximation of bounded functions with values in certain Banach spaces, Math. Ann. 240 (1979), 157-164.

8. C. Olech, Approximation of set-valued functions by continuous functions, Colloq. Math. 19 (1968), 285-293.

9. T.S.S.R.K. Rao, Some remarks on proximinality of higher dual spaces, J. Math. Anal. Appl. 328 (2007), 1173-1177.

10. F.B. Saidi, On the proximinality of the unit ball of proximinal subspaces in Banach spaces: a counterexample, Proc. Amer. Math. Soc. 133 (2005), 2697-2703.

11. D. Yost, Intersecting balls and proximinal subspaces in Banach spaces, Ph.D. thesis, University of Eidenburgh, 1979. 